\title{
Lipid accumulation in hepatocytes induces fibrogenic activation of hepatic stellate cells
}

\author{
Hella Wobser ${ }^{1, *}$, Christoph Dorn ${ }^{1, *}$, Thomas S Weiss ${ }^{2}$, Thomas Amann ${ }^{1}$, Cornelius Bollheimer ${ }^{1}$, Roland Büttner ${ }^{1}$, \\ Jürgen Schölmerich ${ }^{1}$, Claus Hellerbrand ${ }^{1}$ \\ ${ }^{I}$ Department of Internal Medicine I, University of Regensburg, D-93042 Regensburg, Germany; ${ }^{2}$ Center for Liver Cell Research, \\ Department of Surgery, University of Regensburg, D-93042 Regensburg, Germany
}

Despite the initial belief that non-alcoholic fatty liver disease is a benign disorder, it is now recognized that fibrosis progression occurs in a significant number of patients. Furthermore, hepatic steatosis has been identified as a risk factor for the progression of hepatic fibrosis in a wide range of other liver diseases. Here, we established an in vitro model to study the effect of hepatic lipid accumulation on hepatic stellate cells (HSCs), the central mediators of liver fibrogenesis. Primary human hepatocytes were incubated with the saturated fatty acid palmitate to induce intracellular lipid accumulation. Subsequently, human HSCs were incubated with conditioned media (CM) from steatotic or control hepatocytes. Lipid accumulation in hepatocytes induced the release of factors that accelerated the activation and proliferation of HSC, and enhanced their resistance to apoptosis, largely mediated via activation of the PI-3-kinase pathway. Furthermore, CM from steatotic hepatocytes induced the expression of the profibrogenic genes TGF- $\beta$, tissue inhibitor of metallo-proteinase-1 (TIMP-1), TIMP-2 and matrix-metallo-proteinase-2, as well as nuclear-factor $\mathrm{\kappa B}$-dependent MCP-1 expression in HSC. In summary, our in vitro data indicate a potential mechanism for the pathophysiological link between hepatic steatosis and fibrogenesis in vivo. Herewith, this study provides an attractive in vitro model to study the molecular mechanisms of steatosis-induced fibrogenesis, and to identify and test novel targets for antifibrotic therapies in fatty liver disease.

Keywords: fibrosis, hepatic stellate cells, non-alcoholic fatty liver disease, steatosis, in vitro model

Cell Research (2009) 19:996-1005. doi: 10.1038/cr.2009.73; published online 23 June 2009

\section{Introduction}

Non-alcoholic fatty liver disease (NAFLD) has emerged as a considerable public health concern as its major risk factors, that is, obesity and insulin resistance, seem to reach epidemic proportions worldwide. Histological studies of biopsies taken from patients with NAFLD indicate that a significant number of patients progress to

\footnotetext{
*These authors contributed equally to this work. Correspondence: Claus Hellerbrand

Tel: +49-941-944-7155; Fax:+49-941-944-7154

E-mail: claus.hellerbrand@klinik.uni-regensburg.de

Abbreviations: CM (conditioned medium); FFAs (free fatty acids); HSC (hepatic stellate cells); NAFLD (non-alcoholic fatty liver disease); NASH (non-alcoholic steatohepatitis); MMP (matrix-metallo-proteinase); NFKB (nuclear-factor $\mathrm{\kappa B}$ ); $\mathrm{PHH}$ (primary human hepatocytes); TIMP-1/-2 (tissue inhibitor of metallo-proteinase-1/-2)

Received 4 September 2008; revised 2 January 2009; accepted 15 February 2009; published online 23 June 2009
}

non-alcoholic steatohepatitis (NASH) that leads to liver fibrosis and finally cirrhosis [1]. Recent reports about cryptogenic cirrhosis most likely representing "burnedout NASH" further support its potentially progressive nature [2]. Once cirrhosis has developed, the mortality rate increases significantly due to a high risk of hepatic decompensation and hepatocellular carcinoma [3].

According to the two-hit-hypothesis of NASH, hepatic steatosis ("first hit") is a prerequisite for subsequent events ("second hits"), involving environmental and genetic factors that lead to liver injury [4]. Free fatty acids (FFAs) appear to be the major mediators of excessive hepatic lipid accumulation. The rate of hepatic FFA uptake is not regulated, and therefore, is proportional to plasma FFA concentrations [5]. In humans with NAFLD, circulating FFAs are commonly elevated, and their plasma levels correlate with disease severity [6]. Hepatic injury may occur when the capacity of hepatocytes to safely store excess FFA in form of TGs in lipid droplets 
is exceeded. Oxidative stress, mitochondrial dysfunction and upregulation of pro-inflammatory cytokines ("second hits") have been suggested to be major consequences of cellular lipid overload [7-9], potentially contributing to inflammatory liver damage and fibrogenesis in NASH.

Of note, hepatic steatosis has been identified as a risk factor for the progression of hepatic fibrosis in a wide range of liver diseases including alcoholic liver disease, chronic hepatitis $\mathrm{C}$ and hemochromatosis $[10,11]$.

Current evidence indicates that hepatic stellate cells (HSCs) are central mediators of hepatic fibrosis. During hepatic injury, HSCs undergo a phenotypic transformation from a quiescent retinoid-storing cell to a highly proliferative myofibroblast-like celltype, a process termed activation. Activated HSCs express large amounts of extracellular matrix (ECM), including collagen type I, fibronectins and proteoglycans. Deposition of ECM is further enhanced by the production of tissue inhibitors of metalloproteinases (TIMP), which prevent the degradation of ECM, leading to a net accumulation of ECM with a gradual disruption of normal liver architecture. During liver injury, activated HSCs also release a large number of cytokines among which TGF- $\beta$ is considered as the main fibrogenic cytokine. De novo expression of pro-inflammatory cytokines and chemokines further enhances hepatic fibrogenesis by recruiting leukocytes and perpetuating the inflammatory response [12, 13]. Finally, it is also generally assumed that soluble mediators, secreted by hepatocytes, contribute to the stimulation and activation of HSC in chronic liver diseases $[10,14]$.

In order to better understand the pathogenesis of NAFLD/NASH and to identify factors involved in its progression to fibrosis, we aimed to establish an in vitro model to study the molecular mechanisms linking hepatic steatosis to fibrogenesis.

\section{Results}

Palmitate treatment induces cellular lipid accumulation in hepatocytes

Palmitate (C16:0) is the most prevalent long-chain saturated FFA found in circulation. It is bound to albumin, with a physiological ratio of fatty acid to albumin of approximately $2: 1$. In states of insulin resistance and obesity (as major risk factors for NAFLD/NASH), serum fatty acid levels are commonly elevated, yielding ratios as high as 7.5:1 [15].

In order to investigate the effect of hepatic steatosis on HSC, we established a palmitate-induced in vitro fatty liver model. Palmitate was complexed to BSA in a molar ratio of $6.7: 1$, thereby mimicking hyperlipidemic conditions. Exposure to palmitate induced a dose-dependent accumulation of cytosolic lipid droplets in primary human hepatocytes (PHHs), as detected by confocal microscopy after staining with Oil Red O (Figure 1A). These findings were confirmed by colorimetric quantification of the intracellular TG concentration in hepatocytes exposed to 0.1-0.4 mM palmitate (Figure 1B). We found a 2.1 -fold increase $( \pm 0.13 ; P=0.0023)$ in neutral lipid content after incubation with $0.1 \mathrm{mM}$ palmitate compared to BSA-treated control cells. Lipid accumulation was even more pronounced after exposure to 0.2 or $0.4 \mathrm{mM}$ palmitate for $24 \mathrm{~h}(7.3 \pm 0.41$-fold and $11.6 \pm$ 0.6 -fold increase over control).

Measurement of the (residual) palmitate concentration in the supernatant revealed a complete uptake of palmitate by hepatocytes incubated for $24 \mathrm{~h}$ with palmitate at a concentration of 0.1 or $0.2 \mathrm{mM}$. After 24-h incubation
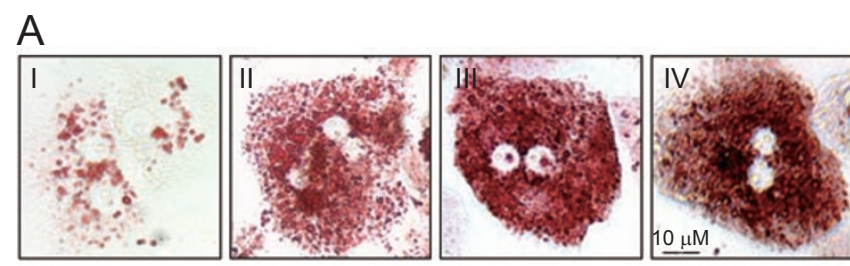

B
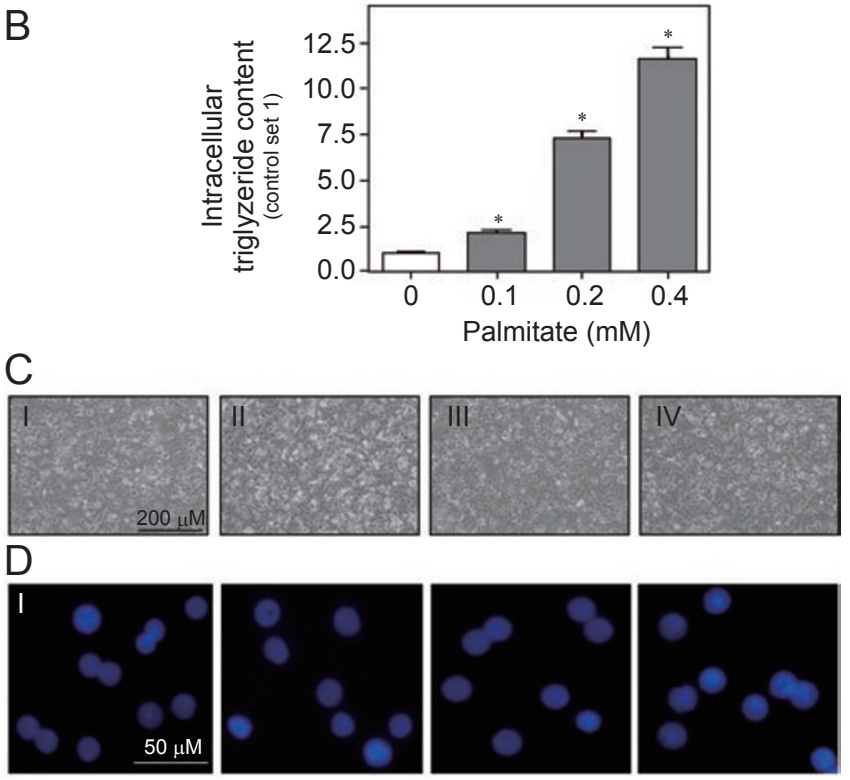

Figure 1 Intracellular lipid accumulation and lack of cytotoxic effects in hepatocytes after stimulation with palmitate. Primary human hepatocytes (PHHs) were incubated with increasing palmitate concentrations (II: $0.1 \mathrm{mM}$, III: $0.2 \mathrm{mM}$, and IV: $0.4 \mathrm{mM}$ ) for $24 \mathrm{~h}$. I: Cells treated with $0.4 \%$ (w/v) FFA-free BSA served as controls. (A) Oil red O staining. (B) Colorimetric assessment of intracellular lipid content. ( ${ }^{\star} P<0.05$ compared to control). (C) Phase-contrast images of $\mathrm{PHH}$ cultures. (D) Chromatin staining (Hoechst) 
with $0.4 \mathrm{mM}$ palmitate, approximately $85 \%$ of the FFA was taken up by the hepatocytes (data not shown).

Viability of PHHs was not affected by the stimulation with increasing doses of palmitate (0.1-0.4 mM) as judged by microscopical analysis (Figure 1C), and the assessment of the release of LDH and transaminases into the culture medium (data not shown). Furthermore, nuclear staining revealed no significant signs of apoptosis (i.e. chromatin condensation and fragmentation) even at the highest palmitate concentration $(0.4 \mathrm{mM}$; Figure 1D).

In contrast to $\mathrm{PHH}$ and similarly as reported by Malhi et al. [16], HepG2 and Huh7 hepatoma cell lines showed signs of lipotoxicity after stimulation with palmitate at concentrations higher than $0.2 \mathrm{mM}$ (data not shown).

Hence, to exclude a direct effect of residual palmitate in the supernatant of steatotic hepatocytes and to allow the potential substitution of PHH by hepatoma cells for the planned in vitro model (see below), we selected palmitate at a concentration of $0.2 \mathrm{mM}$ for the subsequent studies.

Here, we generated conditioned media (CM) from $\mathrm{PHH}$ from the same donor stimulated with either $0.2 \mathrm{mM}$ palmitate or BSA as control. Subsequently, the effects of both CM on HSCs were compared.

Conditioned medium of steatotic hepatocytes accelerates HSC activation and profibrogenic gene expression of HSC

Initially, we aimed to analyze the effect of steatotic hepatocytes on the in vitro activation process of HSC.
A

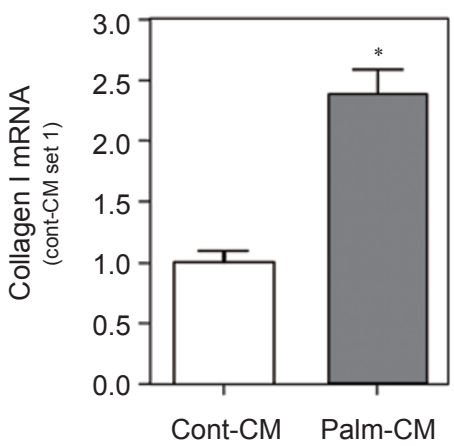

C

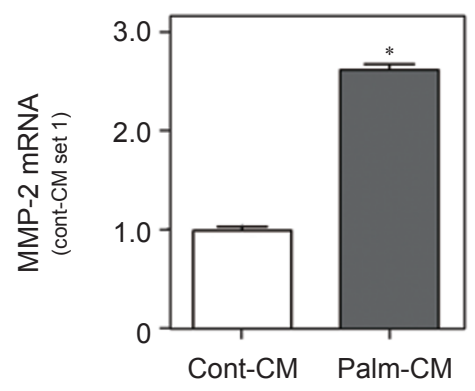

E

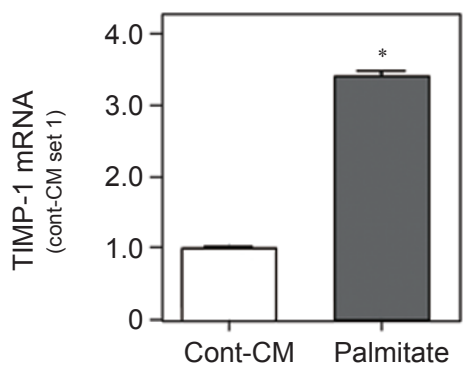

B

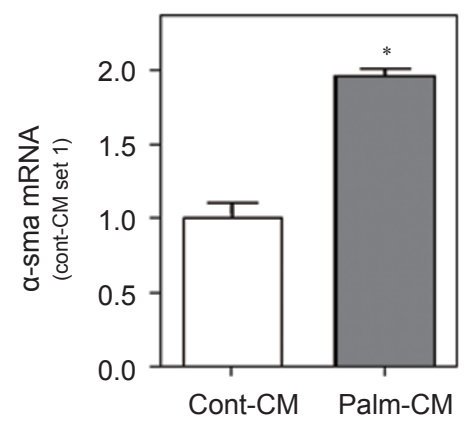

$\mathrm{D}$

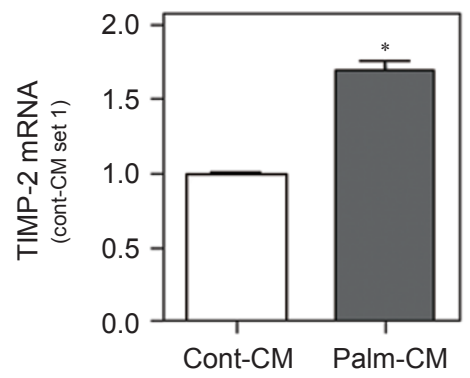

$\mathrm{F}$

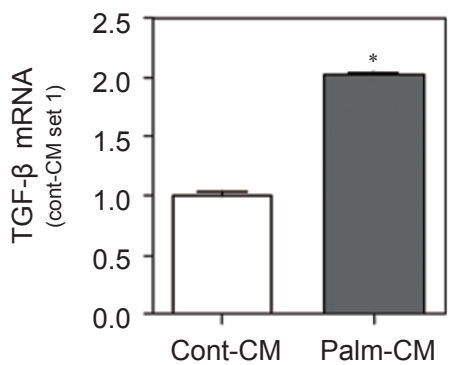

Figure 2 Conditioned medium from steatotic hepatocytes induced activation and profibrogenic gene expression of activated hepatic stellate cells. At 2 days after isolation HSCs were incubated with conditioned medium from palmitate-treated (palmitate) or control hepatocytes for $72 \mathrm{~h}$. Subsequently, (A) collagen I and (B) $\alpha$-sma mRNA expression were analyzed by quantitative PCR analysis. Activated HSCs were incubated with conditioned medium derived from palmitate-treated or control hepatocytes. Subsequently, (C) MMP-2, (D) TIMP-1, (E) TIMP-2 and (F) TGF- $\beta$ mRNA expression were analyzed by quantitative PCR analysis. ( $P<0.05$ compared to control-CM). 
At 2 days after isolation, human HSCs were exposed to $\mathrm{CM}$ from palmitate-treated or control PHH for 3 days. Subsequently, mRNA expression of two established markers of HSC activation, namely collagen type I and alpha-smooth muscle actin ( $\alpha$-sma), was determined by quantitative RT-PCR analysis. HSC exposed to CM from steatotic hepatocytes revealed significantly higher collagen type I $(2.4 \pm 0.2, P=0.025$; Figure $2 \mathrm{~A})$ and $\alpha$-sma $(2.0 \pm 0.1, P=0.014$; Figure $2 \mathrm{~B})$ expression than HSC treated with $\mathrm{CM}$ from control hepatocytes.

Next, we analyzed the effect of CM from steatotic PHH on activated human HSC with regards to the expression of various key fibrosis-related genes. As compared to CM from control hepatocytes, stimulation with $\mathrm{CM}$ from steatotic hepatocytes induced a significantly higher expression of matrix-metallo-proteinase-2 (MMP2) (2.6 \pm 0.1 -fold, $P=0.002$; Figure 2 C), TIMP-1 (3.4 \pm 0.1 -fold, $P=0.0007$; Figure 2E) and TIMP-2 (1.7 \pm 0.1 fold, $P=0.007$ Figure 2D) in activated HSC. Furthermore, TGF- $\beta$ was significantly increased after stimulation with $\mathrm{CM}$ from steatotic hepatocytes as compared to $\mathrm{CM}$ from control cells $(2.0 \pm 0.1$-fold, $P=0.003$; Figure $2 \mathrm{~F})$.

Here as well as in subsequent experiments, it was crucial to demonstrate that soluble factors released by steatotic hepatocytes rather than residual palmitate in the supernatant were responsible for the profibrogenic effects observed in HSC upon stimulation with CM from palmitate-treated hepatocytes. Therefore, we loaded CM derived from steatotic hepatocytes on a 5-kDa cut-off spin column before stimulating HSC. Thus, proteins with a molecular weight above $5 \mathrm{kDa}$ were removed from $\mathrm{CM}$ while potential residual FFAs were not retained by the column. This purified CM was no longer able to induce TGF- $\beta$ or TIMP-1 mRNA expression in HSC (Supplementary information, Figure S1).

Conditioned medium of steatotic hepatocytes enhances HSC proliferation and resistance to apoptosis

To assess the potential mitogenic effect of steatotic hepatocytes on HSC in vitro, proliferation of activated HSC exposed to CM from either palmitate-stimulated or control hepatocytes was compared (Figure 3A). Exposure to $\mathrm{CM}$ from steatotic hepatocytes significantly reduced doubling time as compared to activated HSC stimulated with $\mathrm{CM}$ from control hepatocytes $(5.8 \pm 0.2$ vs. $9.3 \pm 0.9$ days; $P=0.017$ ).

In addition to a high mitogenic activity, activated HSCs are characterized by high resistance to apoptosis. Recent studies indicate that activated human HSCs do not undergo spontaneous apoptosis and survive from well-established pro-apoptotic stimuli like prolonged serum deprivation and exposure to Fas ligand, TNF or
NGF [17]. Resistance of HSC to apoptosis has therefore been proposed to play a key role in the progression of

A

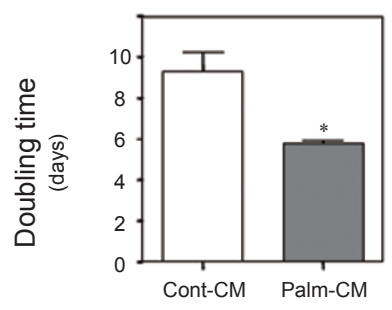

B
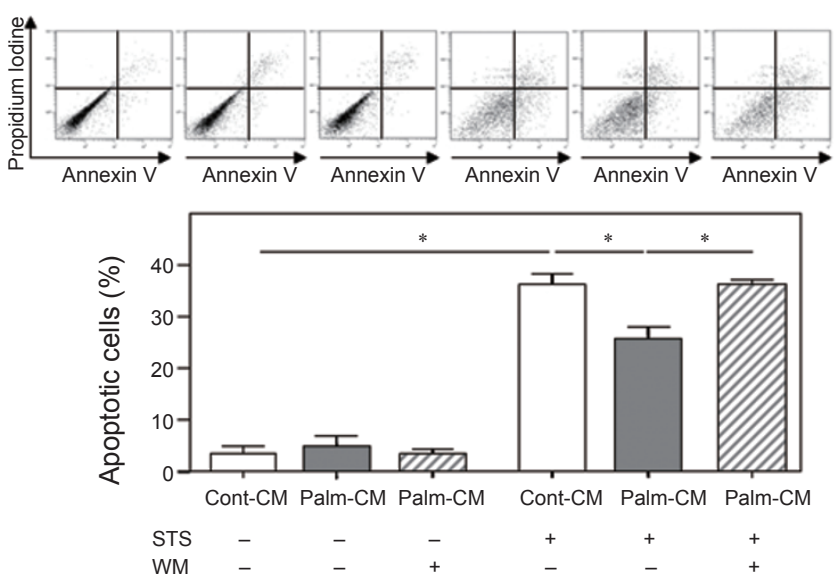

C

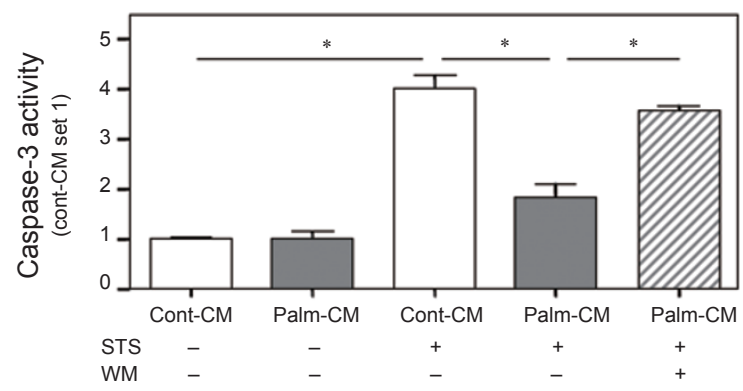

Figure 3 Conditioned medium from steatotic hepatocytes enhanced proliferation and resistance to apoptosis of activated hepatic stellate cells. (A) Doubling time of activated HSC incubated with conditioned medium derived from palmitate-treated or control hepatocytes. ( $P<0.05$ compared to control $\mathrm{CM}$ ). Activated HSCs were pre-incubated for $2 \mathrm{~h}$ with $\mathrm{CM}$ from palmitatetreated or control hepatocytes. Subsequently, apoptosis was induced by staurosporine (STS) treatment (500 nM; 6 h) either in the presence or absence of the PI-3 kinase inhibitor wortmannin (WM; 200 nM). (B) The rate of apoptotic cells was analyzed by flow cytometry (upper panel). Total number of apoptotic cells was determined by calculation of Annexin $\mathrm{V}+$ and PI- cells (reflecting early apoptosis) together with Annexin $\mathrm{V}+$ and $\mathrm{PI}+$ cells (reflecting late apoptosis / secondary necrosis) (lower panel). (C) Caspase-3-like protease activity was measured in cytosolic protein extracts by cleavage of the fluorigenic substrate Z-DEVDRhodamine-110. Activities are represented as fold increase of Rhodamine-110 fluorescence over control. ( $P<0.05)$. 
fibrosis in chronic liver disease.

In order to study the potential anti-apoptotic effect of $\mathrm{CM}$ from steatotic hepatocytes, we used the potent apoptosis inducer staurosporine (STS), a broad-acting kinase inhibitor. HSCs were starved overnight and pre-incubated for $2 \mathrm{~h}$ with $\mathrm{CM}$ collected from either palmitate-treated or control hepatocytes. Subsequently, STS (500 nM) was added and cells were incubated for another $6 \mathrm{~h}$.

As expected, without STS, no significant apoptosis (Annexin-V-positive; PI-negative) was observed in HSC exposed to CM from steatotic or control hepatocytes (3.5 \pm $1.4 \%$ and $3.5 \pm 0.9$; Figure $3 \mathrm{~B}$ ). In contrast, STS treatment induced apoptosis in approximately one-third of control HSC $(36.4 \pm 2.0 \% ; P=0.0002$; Figure 3B). CM collected from steatotic hepatocytes significantly protected HSC from STS-induced apoptosis $(25.7 \pm 4.1 \%$; $P=0.026$; Figure 3B). Of note, the anti-apoptotic effect of $\mathrm{CM}$ from steatotic hepatocytes was abrogated in the presence of the selective PI-3 kinase inhibitor wortmannin $(36.4 \pm 1.1 \%)$, suggesting that the pro-survival effect on activated HSC was largely mediated via activation of the PI-3 kinase signaling.

In line with these data, preincubation of activated HSC with CM from steatotic hepatocytes significantly inhibited STS-induced caspase-3 activation as compared to $\mathrm{CM}$ from control hepatocytes $(1.8 \pm 0.3$-fold vs. $4.0 \pm$ 0.3 -fold, $P=0.02$; Figure 3C). Pharmacological suppression of growth factor survival signaling by administration of wortmannin again abrogated the anti-apoptotic effect of CM from steatotic hepatocytes $(3.6 \pm 0.1$-fold, Figure 3C).

Conditioned medium of steatotic hepatocytes induces $N F-\kappa B$-dependent proinflammatory gene expression in activated hepatic stellate cells

In addition to the expression of pro-fibrogenic genes, $\mathrm{CM}$ from steatotic PHH stimulated pro-inflammatory gene expression in activated HSC. Thus, activated HSC exposed to $\mathrm{CM}$ from palmitate-treated hepatocytes showed significantly higher MCP-1 mRNA $(2.0 \pm 0.03$ fold, $P=0.002$; Figure 4A) and protein (Supplementary information, Figure S2) levels than HSC exposed to CM from control hepatocytes. The expression of MCP-1 in HSC is highly regulated via activation of the transcription factor nuclear-factor $\kappa \mathrm{B}(\mathrm{NF}-\kappa \mathrm{B})$, and accordingly, preincubation with the proteasome inhibitor MG132 (10 $\mu \mathrm{g} / \mathrm{ml}$ ) reduced MCP-1 expression in both HSC stimulated with CM from steatotic and HSC stimulated with CM from control hepatocytes to similar levels (Figure 4A).

In line with these findings, nuclear extracts from HSC stimulated with CM from steatotic hepatocytes showed significantly higher NF- $\mathrm{KB}$ activity than cells incubated with $\mathrm{CM}$ from control hepatocytes $(1.5 \pm 0.1$-fold, $P=$ 0.04; Figure 4B).

Conditioned medium of steatotic hepatoma cells induces profibrogenic effects on an activated hepatic stellate cell line

Our analysis performed with primary human cells suggests in vitro stimulation of HSC by CM from steatotic hepatocytes as a new in vitro model to study steatosisinduced fibrogenesis. However, primary cell cultures of human hepatocytes and HSC do have difficulties because of the limited access, their finite lifespan and the specific culture techniques. Therefore, we analyzed whether the model could be reproduced with hepatoma and HSC lines, as they are available in infinite reproducible quantities. For these experiments we used the hepatoma cell lines HepG2 and HUH-7 and an immortalized non-malignant HSC cell line (hTERT-HSC). The latter has been generated by stable transfection of primary human HSC with human telomerase reverse transcriptase (hTERT)

A

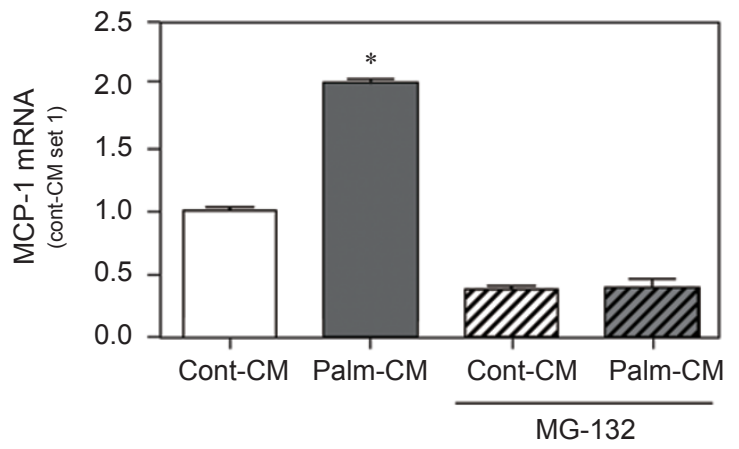

B

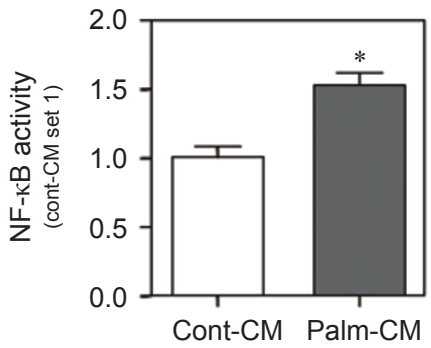

Figure 4 Conditioned medium from steatotic hepatocytes induced MCP-1 expression and NF-kB activity in activated hepatic stellate cells. (A) MCP-1 mRNA expression of activated HSC after incubation with conditioned medium derived from palmitatetreated or control hepatocytes either in the presence or absence of the proteasome inhibitor MG-132 $(10 \mu \mathrm{g} / \mathrm{ml}$; or the solvent DMSO as control). Subsequently, MCP-1 mRNA expression was analyzed by quantitative PCR analysis. (B) NF- $\mathrm{KB}$ activity in nuclear extracts of activated HSC after $2 \mathrm{~h}$ incubation with conditioned medium derived from palmitate-treated or control hepatocytes. ( $P<0.05$ compared to control $\mathrm{CM}$ ). 
and has been previously characterized in detail $[18,19]$.

Similarly as observed in $\mathrm{PHH}$, we found a significant increase in neutral lipid content in hepatoma cells after incubation with $0.2 \mathrm{mM}$ palmitate compared to BSAtreated control cells $(4.3 \pm 0.5$-fold; $P=0.0035$; Figure $5 \mathrm{~A})$.

CM from steatotic HUH7 cells and HepG2 cells (data not shown) induced a significant increase in TGF- $\beta$ (1.7

A

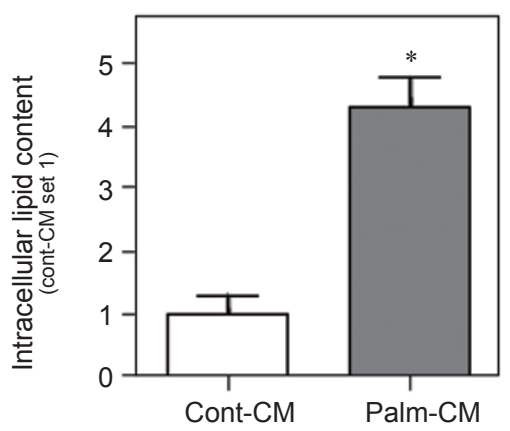

B

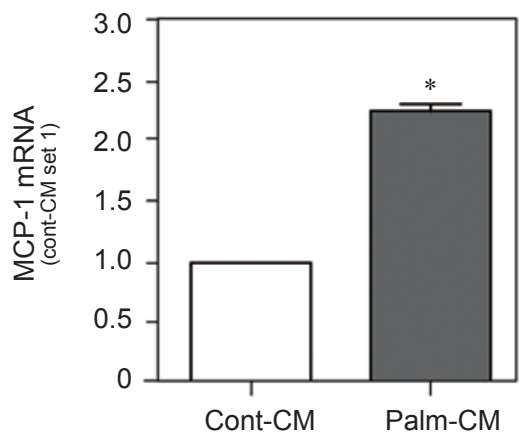

C

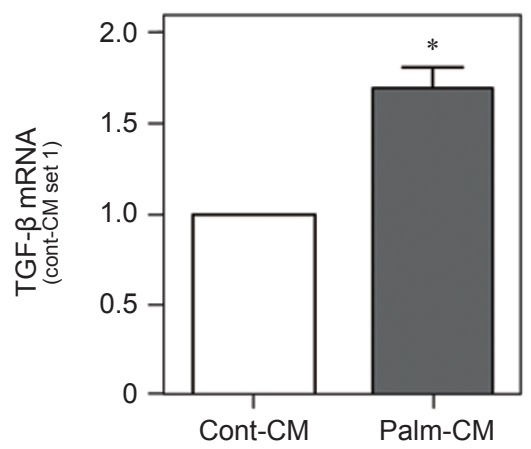

Figure 5 Conditioned medium of steatotic hepatoma cells induced MCP-1 and TGF- $\beta$ expression in an activated hepatic stellate cell line. (A) Intracellular lipid accumulation in Huh7 cells after incubation with palmitate $(0.2 \mathrm{mM}$ for $24 \mathrm{~h})$. (B) MCP-1 and (C) TGF- $\beta$ mRNA expression in an immortalized non-malignant HSC cell line after incubation with conditioned medium derived from palmitate-treated or control Huh7 cells. ( ${ }^{*} P<0.05$ compared to control CM). \pm 0.1 -fold, $P=0.023$; Figure 5B) and MCP-1 (2.3 \pm 0.1 -fold, $P=0.0023$; Figure 5C) mRNA expression in hTERT-HSC. Of note, these effects of steatotic hepatoma cells on hTERT-HSC were comparable to the profibrogenic effects of steatotic PHH on primary HSC (Figure 4A and Figure 2F). In summary, these data indicate that hepatoma and HSC lines are suitable to serve as an in vitro model to study profibrogenic effects of hepatic steatosis.

\section{Discussion}

In the present study, we aimed to establish an in vitro model to investigate the effect of hepatocellular lipid accumulation on HSCs, which play a critical role in hepatic fibrogenesis $[12,13]$.

Until recently, hepatic steatosis was regarded as a benign condition, but increasing evidence suggests that lipid accumulation sensitizes the liver to events that lead to inflammation and fibrosis [4]. In humans, the severity of hepatic steatosis correlates with the stage of liver fibrosis in a wide range of liver diseases. Additionally, weight reduction has been shown to significantly reduce HSC activation and fibrosis [20]. However, it remains unclear if hepatocytic lipid accumulation per se can initiate inflammation and fibrogenesis. Our results strongly suggest that steatotic hepatocytes release soluble factors that enhance pro-inflammatory and profibrogenic gene expression in HSC, and furthermore, induce their activation, proliferation and resistance to apoptosis.

"Activation" of HSC referring to the conversion of HSC from quiescent to highly proliferative and fibrogenic cells is a key event of liver fibrogenesis $[12,13]$. The activation process is classically recognized by the modulation of the cytoskeletal filament, primarily the enhanced expression of contractile $\alpha$-sma filaments [21]. We found a significantly increased expression of $\alpha$-sma in freshly isolated human HSC cultured in CM from steatotic hepatocytes as compared to $\mathrm{CM}$ from control cells. Another key feature of activated HSC is the production of large amounts of collagen type I, the most abundant ECM protein in cirrhotic livers $[12,13]$. Of note, transcript levels of collagen type I showed a significant increase in HSC treated with CM from steatotic hepatocytes. These data clearly indicate that lipid accumulation in hepatocytes induces the secretion of factors accelerating the activation process of HSC.

The observed upregulation of collagen production was accompanied by an increased expression of TIMP-1 and TIMP-2 in activated HSC; both are known inhibitors of matrix degradation. In addition, expression of MMP-2 was upregulated by CM from steatotic hepatocytes. MMP- 
2 is considered as a profibrogenic MMP, as it regulates the degradation of basal lamina allowing replacement by fibrillar collagens during fibrogenesis [13].

Among the numerous profibrogenic mediators, TGF- $\beta$ is regarded as the most potent in liver fibrosis. Overexpression of TGF- $\beta$ in the liver has been shown to induce severe liver fibrosis [22], whereas disrupting its synthesis or signaling pathway markedly decreased fibrosis in experimental models [23]. In HSC, TGF- $\beta$ promotes the activation process, stimulates the synthesis of ECM proteins and inhibits their degradation [24]. In the present study, CM from steatotic hepatocytes induced a pronounced increase of $T G F-\beta$ expression in activated HSC.

Together these findings strongly suggest that hepatic steatosis promotes fibrogenesis by increasing the number of activated HSC through enhanced proliferation, accompanied by a fibrogenic gene expression pattern clearly favoring ECM accumulation.

Activated human HSC have been shown to survive well-established pro-apoptotic stimuli or culture conditions. Caspase-dependent apoptotic cell death of activated human HSC was only observed when protein synthesis or transcription was inhibited by cycloheximide or actinomycin A [17]. In the present study, we provide evidence that inhibition of cell cycle progression by the kinase inhibitor STS is sufficient to reliably induce apoptosis in activated human HSC. It is commonly believed that the observed protection from apoptosis in activated HSC is at least in part mediated via an increased expression of the anti-apoptotic proteins bcl-2 or bcl-xL [17, 25]. In addition, direct interactions with ECM components leading to activation of the FAK/PI-3K-dependent survival pathway [26], and the secretion of TIMP-1 thereby preventing MMP-dependent matrix degradation [27] may promote the survival of activated HSC. Finally, autocrine stimulation by cytokines like TGF- $\beta$ or IGF-1 was regarded as a potential protective mechanism [28, 29]. In the in vitro model presented here, co-incubation of HSC with $\mathrm{CM}$ of steatotic hepatocytes significantly reduced STS-induced apoptotic cell death.

As STS induced apoptosis in HSC efficiently within a few hours, we conclude that the protective effect of $\mathrm{CM}$ from steatotic hepatocytes was caused by pro-survival mediators secreted from hepatocytes rather than autocrine mechanisms originating from HSC. Suppression of apoptosis by $\mathrm{CM}$ from steatotic hepatocytes was lost in the presence of the selective PI-3 kinase inhibitor wortmannin, suggesting that the pro-survival effect of $\mathrm{CM}$ from steatotic hepatocytes was largely mediated via activation of the PI-3 kinase signaling.

PI-3 kinase activation is known to be important for HSC activation, migration, cell attachment, as well as
PDGF-induced cell proliferation and profibrogenic gene expression $[12,13,30]$. Further, we and others have shown that activation of the transcription factor NF- $\mathrm{KB}$ plays a central role in HSC activation, proinflammatory gene expression and resistance to apoptosis $[12,13,31$, 32]. Of note, $\mathrm{CM}$ from steatotic hepatocytes induced both PI3K and NF-kB activity in HSC. Based on these findings, our results provide strong evidence that the release of soluble mediators by steatotic hepatocytes and the concomitant induction of key signaling cascades of fibrogenic activation of HSC play a crucial part in the progression of simple steatosis to NASH and fibrosis.

In addition to PI-3 kinase and NF- $\kappa \mathrm{B}$, several other factors and signaling cascades have been shown to play a crucial role in the activation of HSC and in the associated pathophysiological changes. Thus, AT1 receptors are expressed on activated HSC, which themselves were shown to generate Angiotensin II (Ang II) de novo [12, 33]. Ang II enhances liver fibrosis, while Ang II receptor antagonist (ARB) are capable of inhibiting fibrosis in murine NASH models and patients [34, 35]. Further, growing evidence suggests that peroxisome proliferatoractivated receptors (PPARs) are implicated in NASHinduced fibrosis. Thus, PPAR- $\gamma$ agonists ("glitazones") have been demonstrated to substantially improve liver histology in patients with NASH [36]. It remains to be determined whether ARB or PPAR- $\gamma$ agonists are capable of affecting the profibrogenic effect of $\mathrm{CM}$ from steatotic hepatocytes, but regardless, we would like to propose the idea to study these and other questions in our in vitro model.

Currently, we can only speculate on the profibrogenic factors released by steatotic hepatocytes. However, these factors appear to be larger than $5 \mathrm{kDa}$, since application of 5-kDa cut-off spin columns abrogated the profibrogenic effect of CM. Further, this experiment was crucial to confirm that the residual palmitate in the supernatant does not account for the profibrogenic effects observed in HSC upon stimulation with CM from palmitate-treated hepatocytes. This is further confirmed by a previous study by Abergel et al. [37]. These authors found that palmitic acid significantly decreased HSC proliferation as well as $\alpha$-sma and collagen expression. These findings indicate that potentially remaining palmitate in the $\mathrm{CM}$ of steatotic hepatocytes can be excluded as the cause of profibrogenic effects on HSC, since, if at all, it would exhibit anti-fibrogenic effects.

Interestingly, in preliminary analysis we found increased TGF- $\beta$ and Interleukin- 8 (IL- 8 ) mRNA expression in hepatocytes upon stimulation with palmitate (data not shown). IL-8 is known to be elevated in patients with NAFLD/NASH, and IL-8 levels correlate with disease 
severity [38]. Further and interestingly, Schulze-Krebs et al. [39] have previously shown that TGF- $\beta$ expression was significantly upregulated in Hepatitis $C$ virus (HCV)-replicating human hepatoma cells, and that CM from those HCV-replicating hepatoma cells promoted profibrogenic gene expression in HSC. However and notably, these authors found that TGF- $\beta$ was responsible for only approximately $50 \%$ of the profibrogenic effect observed in their model. We hypothesize that in this previous study and in our model several different factors contribute to the fibrogenic effects on HSC.

In summary, we established an attractive new in vitro model to study molecular mechanisms of steatosis-induced hepatic fibrogenesis. The present data demonstrate that steatotic hepatocytes release soluble factors that promote activation, proliferation and apoptosis resistance of human HSC. Furthermore, these soluble mediators enhanced pro-inflammatory and pro-fibrogenic gene expression in activated HSC. The precise mechanism connecting hepatic steatosis to the hepatic release of yetunknown profibrotic mediators has to be addressed in further studies. The in vitro model presented here may help to elucidate the pathophysiological mechanisms driving simple hepatic steatosis to NASH and to establish novel anti-inflammatory and anti-fibrotic therapeutic strategies in steatosis-related liver disease.

\section{Materials and Methods}

\section{Cell isolation and cell culture}

Human liver tissue for cell isolation was obtained from the charitable state controlled foundation HTCR, with the informed patient's consent.

Isolation and culture of PHHs and HSC were performed as described previously [40-42]. The isolation procedure and cell culture on uncoated tissue culture dishes led to the activation of HSC as described $[40,43]$.

HepG2 (ATCC HB-8065) and HuH-7 (JCR B0403) cells were cultured as described [41, 42]. Furthermore, we used an immortalized activated human HSC line generated by ectopic expression of hTERT that has been characterized before $[18,19]$.

\section{Fatty acid treatment and preparation of conditioned me- dium}

Palmitic acid (Cat \# P 0500) was obtained from Sigma pharmaceuticals (Hamburg, Germany). Preparation of the palmitate stock solution was carried out as described previously [44]. Briefly, a $100 \mathrm{mM}$ palmitate stock solution was prepared in $0.1 \mathrm{mM} \mathrm{NaOH}$ by heating at $70{ }^{\circ} \mathrm{C}$. A $10 \%$ (w/v) FFA-free BSA (Sigma) solution was prepared in $\mathrm{ddH}_{2} \mathrm{O}$ and maintained at $55^{\circ} \mathrm{C}$ in a water bath. $10 \mathrm{mM}$ FFA/1\% BSA solution was obtained by complexing the appropriate amount of palmitate stock solution to $10 \%$ BSA at $55^{\circ} \mathrm{C}$ for another $30 \mathrm{~min}$. The above solution was then cooled to $25^{\circ} \mathrm{C}$, filter sterilized and stored at $-20^{\circ} \mathrm{C}$ until use.

$\mathrm{PHH}$ and hepatoma cells were grown in DMEM supplemented with penicillin $(400 \mathrm{U} / \mathrm{ml})$, streptomycin $(50 \mu \mathrm{g} / \mathrm{ml})$, L-glutamine $(300 \mu \mathrm{g} / \mathrm{ml}), 0.2 \% \mathrm{FCS}$ and palmitate in different concentrations as indicated. In total, $0.4 \%(\mathrm{w} / \mathrm{v})$ FFA-free-BSA-treated cells served as controls. After $24 \mathrm{~h}, \mathrm{CM}$ was clarified by centrifugation at $6000 \mathrm{~g}$ to remove cell debris, sterile filtered $(0.45 \mu \mathrm{m}$ pore size membrane filter), and stored in aliquots at $-20{ }^{\circ} \mathrm{C}$ until use.

HSCs were incubated with $\mathrm{CM}$ from palmitate-treated $\mathrm{PHH}$ and hepatoma cells, or CM from control cells for the indicated times. In initial experiments, the expression of profibrogenic genes and proliferation, respectively, in control HSC and HSC treated with $\mathrm{CM}$ from control hepatocytes did not reveal significant differences (data not shown). This finding was in accordance with previous reports [39]. Therefore, in the present study we focused on the comparison between HSC treated with CM from control hepatocytes and steatotic hepatocytes.

For defined experiments, CM was run on 5-kDa cut-off spin columns according to the manufacturer's instructions (Sartorius, Göttingen, Germany) before being used in stimulation experiments.

\section{Oil Red and Hoechst staining}

Palmitate-treated and control cells were washed with cold PBS and fixed in $3.7 \%$ paraformaldehyde at $37{ }^{\circ} \mathrm{C}$ for $15 \mathrm{~min}$ at room temperature, then permeabilized by treatment with a 19:1 mixture of ethanol/acetic acid at $-20{ }^{\circ} \mathrm{C}$ for $15 \mathrm{~min}$. Subsequently, cells were incubated with Oil Red O (Sigma) in PBS for 5min at room temperature. Intracellular lipid accumulation was examined by fluorescence microscopy. To observe nuclear changes indicative of apoptosis, the chromatin-specific dye Hoechst 33258 (Sigma) was used. Here, cells were stained with $1 \mu \mathrm{g} / \mathrm{ml}$ Hoechst 33258 in PBS at room temperature for $20 \mathrm{~min}$.

\section{Intracellular triglyceride assay}

Total TGs were extracted using the method of Bligh and Dyer with slight modifications [45]. In brief, hepatocytes were plated into T25 flasks. At $24 \mathrm{~h}$ after treatment cells were washed with PBS, trypsinized, centrifuged and washed twice in 10\% FFA-free BSA. Cell pellets were weighed into $1 \mathrm{ml}$ of a chloroform-methanol mix $(2: 1 \mathrm{v} / \mathrm{v})$ and incubated for $1 \mathrm{~h}$ at room temperature with occasional shaking to extract the lipid. After addition of $200 \mu 1$ $\mathrm{H}_{2} \mathrm{O}$, vortexing and centrifugation for $5 \mathrm{~min}$ at $3000 \mathrm{~g}$, the lower lipid phase was collected and dried at room temperature. The lipid pellet was re-dissolved in $60 \mu \mathrm{l}$ tert-butanol and $40 \mu \mathrm{l}$ of a Triton $\mathrm{X}$-114-methanol $(2: 1 \mathrm{v} / \mathrm{v}) \mathrm{mix}$, and TGs were measured by means of the GPO-triglyceride kit (Sigma) using appropriate triglyceride standards (Sigma).

\section{Expression analysis}

Isolation of total cellular RNA from cultured cells and tissues and reverse transcription were performed as described previously [40]. Quantitative real-time PCR was performed with specific sets of primers (Table 1) applying LightCycler technology (Roche, Mannheim, Germany) as described [40].

MCP-1 concentration in the supernatant was analyzed by an enzyme-linked immunosorbent assay (ELISA) following the instructor's manual (Biosource, Camarillo, CA, USA).

Analysis of apoptosis by Annexin/PI-based flow cytometry

For detection of apoptosis, cells were stained simultaneously 
Table 1 Sets of primers used for quantitative PCR analysis

\begin{tabular}{lll}
\hline Gene & Forward primer & Reverse primer \\
\hline$\beta$-actin & 5'-CTACGTCGCCCTGGACTTCGAGC & 5'-GATGGAGCCGCCGATCCACACGG \\
Collagen I & 5'-CGGCTCCTGCTCCTCTT & 5'-GGGGCAGTTCTTGGTCTC \\
$\alpha$-sma & 5'-CGTGGCTATTCCTTCGTTAC & 5'-TGCCAGCAGACTCCATCC \\
TIMP-1 & 5'-CCAGAAGTCAACCAGACCACC & 5'-GGGCAGGATTCAGGCTATCTG \\
TIMP-2 & 5'-AGAGGATCCAGTATGAGATCAAGCAG & 5'-TGGTACCTGTGGTTCAGGCTCTTC \\
TGF- $\beta$ & 5'-TTCCCTCGAGGCCCTCCTA & 5'-GCCGCAGCTTGGACAGGATC \\
MMP-2 & 5'-GCTGGGAGCATGGCGATGGATACC & 5'-GGACAGAAGCCGTACTTGCCATCC \\
MCP-1 & 5'-CGCGAGCTATAGAAGAATCAC & 5'-TTGGGTTGTGGAGTGAGTGT \\
\hline
\end{tabular}

with FITC-conjugated Annexin V and propidium iodide (both from Pharmingen, Germany) as described [13]. Analysis of data was performed using the software WinMDI (Version 2.8, http:// facs.scripps.edu).

Analysis of caspase-3 activity

The Apo-One Homogeneous Caspase-3/7 Assay (Promega, Madison, WI, USA) was used to analyze caspase-3 activity in HSC according to the manufacturer's instructions.

Quantification of activated nuclear $N F-\kappa B$ concentration

NF- $\kappa \mathrm{B}$ was quantified in nuclear extracts with the ELISA-based kit TransAm from Active Motif (Rixensart, Belgium) according to the manufacturer's instructions as described [46].

\section{Proliferation assay}

Cell proliferation was measured using the XTT assay (Roche) as described [41, 42].

\section{Statistical analysis}

Values are presented as means \pm SEM. Comparison between groups was made using the Student's unpaired $t$-test. Welch's correction was performed when required. $P<0.05$ was considered statistically significant. All calculations were performed using the statistical computer package GraphPad Prism version 4.00 for Windows (GraphPad Software, San Diego, CA, USA).

\section{Acknowledgments}

We would like to thank Elena Spacenko and Monika Artinger (Department of Internal Medicine I, University of Regensburg, Germany) for excellent technical assistance. This work was supported by grants from the German Research Association (He 2458/14-1 and Schn 620/3-1) and the Medical Faculty of the University of Regensburg (ReForM) to Hella Wobser, Thomas Weiss, and Claus Hellerbrand.

\section{References}

1 Powell EE, Cooksley WG, Hanson R, Searle J, et al. The natural history of nonalcoholic steatohepatitis: a follow-up study of forty-two patients for up to 21 years. Hepatology 1990, 11:74-80.

2 Caldwell SH, Oelsner DH, Iezzoni JC, Hespenheide EE, et al.
Clinical characterization and risk factors for underlying disease. Hepatology 1999, 29:664-669.

3 Ratziu V, Bonyhay L, Di MV, Charlotte F, et al. Survival, liver failure, and hepatocellular carcinoma in obesity-related cryptogenic cirrhosis. Hepatology 2002, 35:1485-1493.

4 Day CP, James OF. Steatohepatitis. a tale of two "hits"? Gastroenterology 1998, 114:842-845.

5 Teli MR, James OF, Burt AD, Bennett MK, Day CP. The natural history of nonalcoholic fatty liver: a follow-up study. Hepatology 1995, 22:1714-1719.

6 Nehra V, Angulo P, Buchman AL, Lindor KD. Nutritional and metabolic considerations in the etiology of nonalcoholic steatohepatitis. Dig Dis Sci 2001, 46:2347-2352.

7 Seki S, Kitada T, Yamada T, Sakaguchi H, et al. In situ detection of lipid peroxidation and oxidative DNA damage in nonalcoholic fatty liver diseases. J Hepatol 2002, 37:56-62.

8 Caldwell SH, Swerdlow RH, Khan EM, Iezzoni JC, et al. Mitochondrial abnormalities in non-alcoholic steatohepatitis. $J$ Hepatol 1999, 31:430-434.

9 Perez-Carreras M, Del Hoyo P, Martin MA, Rubio JC, et al. Defective hepatic mitochondrial respiratory chain in patients with nonalcoholic steatohepatitis. Hepatology 2003, 38:9991007.

10 Reeves HL, Burt AD, Wood S, Day CP. Hepatic stellate cell activation occurs in the absence of hepatitis in alcoholic liver disease and correlates with the severity of steatosis. J Hepatol 1996, 25:677-683.

11 Wahren J, Sato Y, Ostman J, Hagenfeldt L, Felig P. Turnover and splanchnic metabolism of free fatty acids and ketones in insulin-dependent diabetics at rest and in response to exercise. J Clin Invest 1984, 73:1367-1376.

12 Bataller R, Brenner DA. Liver fibrosis. J Clin Invest 2005, 115:209-218.

13 Friedman SL. Mechanisms of disease: Mechanisms of hepatic fibrosis and therapeutic implications. Nat Clin Pract Gastroenterol Hepatol 2004, 1:98-105.

14 Tavill AS. Intracellular pathways of protein synthesis and secretion in the hepatocyte. Semin Liver Dis 1985, 5:95-109.

15 Kleinfeld AM, Prothro D, Brown DL, Davis RC, et al. Increases in serum unbound free fatty acid levels following coronary angioplasty. Am J Cardiol 1996, 78:1350-1354.

16 Malhi H, Bronk SF, Werneburg NW, Gores GJ. Free fatty acids induce JNK-dependent hepatocyte lipoapoptosis. $J$ Biol Chem 2006, 281:12093-12101. 
17 Novo E, Marra F, Zamara E, Valfre dB, et al. Overexpression of Bcl-2 by activated human hepatic stellate cells: resistance to apoptosis as a mechanism of progressive hepatic fibrogenesis in humans. Gut 2006, 55:1174-1182.

18 Schnabl B, Choi YH, Olsen JC, Hagedorn CH, Brenner DA. Immortal activated human hepatic stellate cells generated by ectopic telomerase expression. Lab Invest 2002, 82:323-333.

19 Schnabl B, Purbeck CA, Choi YH, Hagedorn CH, Brenner D. Replicative senescence of activated human hepatic stellate cells is accompanied by a pronounced inflammatory but less fibrogenic phenotype. Hepatology 2003, 37:653-664.

20 Hickman IJ, Clouston AD, Macdonald GA, Purdie DM, et al. Effect of weight reduction on liver histology and biochemistry in patients with chronic hepatitis C. Gut 2002, 51:89-94.

21 Rockey DC, Boyles JK, Gabbiani G, Friedman SL. Rat hepatic lipocytes express smooth muscle actin upon activation in vivo and in culture. J Submicrosc Cytol Pathol 1992, 24:193203.

22 Ueberham E, Low R, Ueberham U, Schonig K, et al. Conditional tetracycline-regulated expression of TGF-beta1 in liver of transgenic mice leads to reversible intermediary fibrosis. Hepatology 2003, 37:1067-1078.

23 Qi Z, Atsuchi N, Ooshima A, Takeshita A, Ueno H. Blockade of type beta transforming growth factor signaling prevents liver fibrosis and dysfunction in the rat. Proc Natl Acad Sci U S A 1999, 96:2345-2349.

24 Hellerbrand C, Stefanovic B, Giordano F, Burchardt ER, Brenner DA. The role of TGFbeta1 in initiating hepatic stellate cell activation in vivo. J Hepatol 1999, 30:77-87.

25 Schnabl B, Purbeck CA, Choi YH, Hagedorn CH, Brenner D. Replicative senescence of activated human hepatic stellate cells is accompanied by a pronounced inflammatory but less fibrogenic phenotype. Hepatology 2003, 37:653-664.

26 Reif S, Lang A, Lindquist JN, Yata Y, et al. The role of focal adhesion kinase-phosphatidylinositol 3-kinase-akt signaling in hepatic stellate cell proliferation and type I collagen expression. J Biol Chem 2003, 278:8083-8090.

27 Murphy FR, Issa R, Zhou X, Ratnarajah S, et al. Inhibition of apoptosis of activated hepatic stellate cells by tissue inhibitor of metalloproteinase-1 is mediated via effects on matrix metalloproteinase inhibition: implications for reversibility of liver fibrosis. J Biol Chem 2002, 277:11069-11076.

28 Saile B, Matthes N, Knittel T, Ramadori G. Transforming growth factor beta and tumor necrosis factor alpha inhibit both apoptosis and proliferation of activated rat hepatic stellate cells. Hepatology 1999, 30:196-202.

29 Issa R, Williams E, Trim N, Kendall T, et al. Apoptosis of hepatic stellate cells: involvement in resolution of biliary fibrosis and regulation by soluble growth factors. Gut 2001, 48:548-557.

30 Reif S, Lang A, Lindquist JN, Yata Y, et al. The role of focal adhesion kinase-phosphatidylinositol 3-kinase-akt signaling in hepatic stellate cell proliferation and type I collagen expression. J Biol Chem 2003, 278:8083-8090.

31 Hellerbrand C, Jobin C, Licato LL, Sartor RB, Brenner DA. Cytokines induce NF-kappaB in activated but not in quiescent rat hepatic stellate cells. Am J Physiol 1998, 275:G269-G278.
32 Hellerbrand C, Jobin C, Iimuro Y, Licato L, et al. Inhibition of NFkappaB in activated rat hepatic stellate cells by proteasome inhibitors and an IkappaB super-repressor. Hepatology 1998, 27:1285-1295.

33 Yoshiji H, Kuriyama S, Yoshii J, Ikenaka Y, et al. Angiotensin-II type 1 receptor interaction is a major regulator for liver fibrosis development in rats. Hepatology 2001, 34:745750.

34 Yokohama S, Yoneda M, Haneda M, Okamoto S, et al. Therapeutic efficacy of an angiotensin II receptor antagonist in patients with nonalcoholic steatohepatitis. Hepatology 2004, 40:1222-1225.

35 Hirose A, Ono M, Saibara T, Nozaki Y, et al. Angiotensin II type 1 receptor blocker inhibits fibrosis in rat nonalcoholic steatohepatitis. Hepatology 2007, 45:1375-1381.

36 Lutchman G, Modi A, Kleiner DE, Promrat K, et al. The effects of discontinuing pioglitazone in patients with nonalcoholic steatohepatitis. Hepatology 2007, 46:424-429.

37 Abergel A, Sapin V, Dif N, Chassard C, et al. Growth arrest and decrease of alpha-SMA and type I collagen expression by palmitic acid in the rat hepatic stellate cell line PAV-1. Dig Dis Sci 2006, 51:986-995.

38 Kugelmas M, Hill DB, Vivian B, Marsano L, McClain CJ. Cytokines and NASH: a pilot study of the effects of lifestyle modification and vitamin E. Hepatology 2003, 38:413-419.

39 Schulze-Krebs A, Preimel D, Popov Y, Bartenschlager R, et al. Hepatitis $\mathrm{C}$ virus-replicating hepatocytes induce fibrogenic activation of hepatic stellate cells. Gastroenterology 2005, 129:246-258.

40 Muhlbauer M, Bosserhoff AK, Hartmann A, Thasler WE, et al. A novel MCP-1 gene polymorphism is associated with hepatic MCP-1 expression and severity of HCV-related liver disease. Gastroenterology 2003, 125:1085-1093.

41 Hellerbrand C, Amann T, Schlegel J, Wild P, et al. The novel gene MIA2 acts as a tumour suppressor in hepatocellular carcinoma. Gut 2008, 57:243-251.

42 Hellerbrand C, Bumes E, Bataille F, Dietmaier W, et al. Reduced expression of CYLD in human colon and hepatocellular carcinomas. Carcinogenesis 2007, 28:21-27.

43 Muhlbauer M, Fleck M, Schutz C, Weiss T, et al. PD-L1 is induced in hepatocytes by viral infection and by interferonalpha and -gamma and mediates $\mathrm{T}$ cell apoptosis. $J$ Hepatol 2006, 45:520-528.

44 Cousin SP, Hugl SR, Wrede CE, Kajio H, et al. Free fatty acid-induced inhibition of glucose and insulin-like growth factor I-induced deoxyribonucleic acid synthesis in the pancreatic beta-cell line INS-1. Endocrinology 2001, 142:229-240.

45 Buettner R, Newgard CB, Rhodes CJ, O'Doherty RM. Correction of diet-induced hyperglycemia, hyperinsulinemia, and skeletal muscle insulin resistance by moderate hyperleptinemia. Am J Physiol Endocrinol Metab 2000, 278:E563-E569.

46 Muhlbauer M, Allard B, Bosserhoff AK, Kiessling S, et al. Differential effects of deoxycholic acid and taurodeoxycholic acid on NF-kappa B signal transduction and IL-8 gene expression in colonic epithelial cells. Am J Physiol Gastrointest Liver Physiol 2004, 286:G1000-G1008.

(Supplementary information is linked to the online version of the paper on the Cell Research website.) 